\title{
Dengue en Colombia: diez años de evolución
}

\author{
Juan Camilo Castrillón, Jhon Carlos Castaño y Silvio Urcuqui
}

\begin{abstract}
Universidad de Antioquia, Medellín Colombia. Grupo Inmunovirología (JCC, SU). Universidad del Quindío, Armenía, Colombia. Grupo Inmunología Molecular (JhCC).

Los autores manifiestan que no existe, de manera directa

o indirecta, ningún tipo de conflicto de intereses, financieros, académicos ni personales para la publicación de este artículo. Este trabajo fue financiado por Colciencias, proyecto № 111551928777 y Banco de la República (Colombia), proyecto $N^{\circ} 2686$

Recibido: 4 de agosto de 2014 Aceptado: 14 de diciembre de

Correspondencia a: Juan Camilo Castrillón Betancur castricore@hotmail.com
\end{abstract}

\section{Introducción}

S e ha sugerido que la epidemia de dengue comenzó en algunas regiones de Asia y el Pacífico, durante y después de la segunda guerra mundial ${ }^{1}$. Los cambios ecológicos que ocurrieron durante dicha época, probablemente favorecieron la expansión geográfica del vector del dengue virus (DENV); además, el alto número de individuos susceptibles (poblaciones locales y soldados) y su alta movilidad, posiblemente crearon las condiciones necesarias para la dispersión del DENV ${ }^{1}$. En América, el dengue comenzó a ser considerado como un problema importante de salud pública sólo hasta hace unas cuantas décadas ${ }^{2}$. En 1950 se inició un programa que tenía como objetivo erradicar el mosquito Aedes aegypti, principal vector del DENV y aunque este programa, coordinado por la Organización Panamericana de la Salud (OPS) fue exitoso en muchos países, el mosquito no pudo erradicarse por completo de toda la región ${ }^{3}$. A finales de la década de 1970, consecuencia de la disminución del apoyo económico para la vigilancia y el control del mosquito, en la mayoría de países de la región tropical de América se volvió a incrementar la población del vector $^{3}$. Como resultado, además de la emergencia de nuevos serotipos virales, conocimos la mayor epidemia de dengue hemorrágico (DH) en toda la región ${ }^{4}$. En 1981, la primera y mayor epidemia de dengue en América ocurrió en Cuba, con aproximadamente 334.000 pacientes con dengue; de ellos, 116.000 fueron hospitalizados y 10.000 progresaron a $\mathrm{DH}^{4}$. Asimismo, una actividad epidémica sin precedentes se produjo en el continente americano en
2001, con más de 609.000 casos de dengue reportados 5 .

Actualmente, el dengue es considerado como una de las enfermedades más importantes transmitidas por mosquitos y uno de los principales problemas de salud pública en el mundo ${ }^{6}$. En los últimos cincuenta años, el número de casos de dengue, tanto en la forma leve como grave [dengue clásico (DC) y dengue hemorrágico (DH)] y la incidencia de la infección por el DENV, ha incrementado de manera alarmante ${ }^{6}$. Se estima que cerca de 2.500 millones de personas distribuidas en más de cien países están en riesgo de contraer la infección y se ha reportado hasta 50 millones de infecciones anuales, de las cuales, 500.000 son casos de DH con 22.000 muertes, principalmente niños ${ }^{7}$.

En el presente estudio se pretende analizar, por año y por regiones, la incidencia y el número de casos de dengue reportados en Colombia en los últimos diez años (2004-2013). Este estudio retrospectivo, muestra cuál es el comportamiento temporal de la enfermedad, los períodos de máxima transmisión y regiones con mayor incidencia y número de casos, y los posibles factores de riesgo para su transmisión. Este análisis podría brindar información muy interesante y de gran utilidad para el establecimiento de estrategias de control del mosquito y de la enfermedad, en las regiones de mayor incidencia en el país.

\section{Material y Métodos}

Entre noviembre de 2012 y marzo de 2013, se realizó una búsqueda de los estudios epidemiológicos y un análisis retrospectivo de los casos de dengue registrados 
en los últimos diez años en Colombia, entre los años 2004 y 2013. Para efectos del presente trabajo, se seleccionó todos los estudios de casos e incidencia de dengue reportados en los registros de la Organización Mundial de la Salud $(\mathrm{OMS})^{8}$, de la OPS, a través del Sistema de Vigilancia en Salud Pública (SIVIGILA) ${ }^{9}$ y del Instituto Nacional de Salud (INS) ${ }^{10}$ de Colombia. Se tomaron los datos de casos de dengue clásico y dengue hemorrágico de las bases de datos de enfermedades transmitidas por vectores y de dengue y dengue grave de los casos reportados a partir de 2010 debido al cambio de la clasificación reportado para los síntomas de dengue por la OMS. La búsqueda de otros estudios epidemiológicos se hizo a través de la herramienta de búsqueda de información PubMed y Google Scholar. Las variables meteorológicas se obtuvieron de la página del Instituto de Hidrología, Meteorología y Estudios Ambientales (IDEAM) ${ }^{11}$ de Colombia y su correlación con la incidencia de dengue en Colombia se halló por medio del método de correlación de Pearson, utilizando la herramienta GraphPad Prism, Version 6.01.

\section{Resultados}

Entre los años 2001 y 2007 se reportó un total de 4.332.731 casos de dengue en aproximadamente 30 países de América. El número de casos de DH para el mismo período fue de $106.037 \mathrm{y}$ el número total de muertes por dengue fue de 1.299, con una tasa de mortalidad de 1,2\%. En Bolivia, Colombia, Ecuador, Perú y Venezuela se reportó el mayor número de casos de $\mathrm{DH}$, con $58 \%$ (61.341 casos) de todos los casos reportados en América en dicho período. Colombia ocupó el primer puesto con el mayor número de muertes por dengue ( 225 casos). Vale la pena resaltar que antes de 1981, el DH era una enfermedad inusual en América. Desde 1981 el dengue es considerado como uno de los problemas de salud pública más importantes no sólo de nuestra región, sino del mundo. Sin embargo, la mayoría de casos de $\mathrm{DH}$ se han reportado en los países asiáticos, donde es la principal causa de hospitalización y muerte en niños.

El 2004 constituye el año con menos casos de DC reportados en el territorio colombiano, con un total de 27.523 (Figura 1). Es decir, se presentó una disminución de $60 \%$, si se compara con los casos reportados en el año 2002. Los departamentos que más casos de DC reportaron fueron Huila, Santander y Tolima, con 3.210, 3.125 y 2.695 casos; respectivamente. Igualmente fue uno de los años con menor número de casos de $\mathrm{DH}$; sólo se reportaron 2.815, siendo Huila, con 1.135 casos (se observó un fuerte incremento, de 596 casos en el 2003, a 1.135) y Santander, con 681 casos. Quindío sigue siendo uno de los departamentos con mayor incidencia de DC con 378,69 casos/100.000 hbts., mientras que Casanare, Guaviare y Huila presentaron tasas mayores a 500 casos/ $100.000 \mathrm{hbts}$. Este último, también presentó la mayor incidencia de DH con 197,66 casos/100.000 hbts., seguido de Guaviare con 87,88 casos/ 100.000 hbts.

Durante el año 2005 se notificaron 43.113 casos de dengue (Figura 1), de los cuales 90\% (38.827 casos) corresponden a DC. En los departamentos en los cuales se registró una disminución de casos, al compararlo con el año 2004, fueron Antioquia, Caldas, Huila y Norte de Santander. Quindío con 4.285 casos, Valle del Cauca con 3.925, Santander con 3.880 y Meta con 3.565 casos, fueron los departamentos con mayor número de casos reportados. Igualmente, Santander, Huila y Valle del Cauca, reportaron el mayor número de casos de DH. En el 2005, la mayor tasa de incidencia (más de 200 casos/100.000 hbts.) se registró en los departamentos de Arauca, Caquetá, Casanare, Cesar, Cundinamarca, Guaviare, Huila, Meta, Quindío, Risaralda, Santander, Tolima y Guainía (donde no se había registrado transmisión y para este año se reportaron 200 casos de DC). Un aspecto para resaltar, es que el 2005 se reportó por primera vez la circulación del DENV serotipo 1 (DENV 1) y la presencia del vector A. aegypti en ciertas regiones donde no se había descrito previamente. Igualmente durante dicho período se observó un pico muy alto de casos de DH en el Departamento de Santander, con un aumento de más de $300 \%$, con respecto al año 2004.

Para el año 2006 se notificaron al SIVIGILA 38.524 casos de dengue (Figura 1); 86\% de DC (33.116 casos) y $14 \%$ de DH (5.408 casos). Comparado con el año 2005, los casos de dengue reportados en el país disminuyeron en $10 \%$. El $50 \%$ de los casos de DC provienen de los depar-

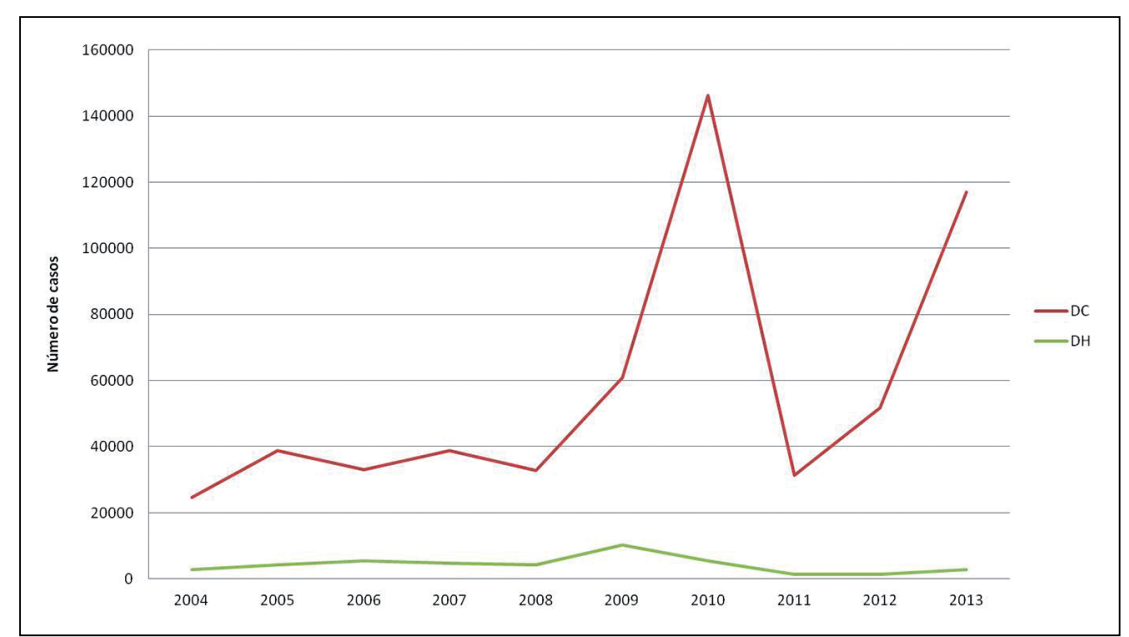

Figura 1. Reporte de casos de dengue clásico y dengue hemorrágico en Colombia entre el 20042013. La curva se realizó con base en la información obtenida de la base de datos del SIVIGILA y del INS de Colombia. 
tamentos de Norte de Santander con 4.207, Santander con 3.923, Casanare con 2.178, Atlántico con 2.097, Antioquia con 2.090 , Risaralda con 1.800 y Sucre con 1.735 casos. Sin embargo, la incidencia de DC para este año fue mayor en los departamentos de Vichada (que presentó un gran incremento de casos, 340, ya que se constituía como uno de los departamentos que menor número de casos presentaba de dengue en el país), Guaviare y Arauca; con $2.143,08 ; 2.009,61$ y 653,35 casos $/ 100.000$ hbts., respectivamente. Los departamentos con mayores casos de DH fueron Santander con 2.645 casos (que corresponden a casi el 50\% de los casos de DH reportados en todo el país), Norte de Santander con 787 y Huila con 368 casos. Los dos primeros departamentos reportan la incidencia de $\mathrm{DH}$ más altas, 176,78 y 97,15/100.000 hbts., respectivamente.

El acumulado de casos notificados al SIVIGILA durante el año 2007 fue de 43.541 casos de dengue (Figura $1) ; 89 \%$ corresponden a DC (38.895 casos) y $11 \%$ a DH (4.646 casos). Al compararlo con lo reportado en el 2006, se observa un incremento de $12 \%$ de los casos de dengue en el país. El $75 \%$ de los casos de dengue reportados proceden de los departamentos de Norte de Santander con 3.572 casos, Antioquia con 3.208, Valle del Cauca con 3.178 , Santander con 3.126, Meta con 2.443 y Casanare con 2.132 casos. Aunque se observó una disminución en el número de casos de DH reportados, al compararlo con el 2006, Santander sigue en primer lugar con 1.330 casos; le sigue Valle del Cauca con 552, Norte de Santander con 531 y Huila con 344. De la misma forma, Santander posee una de las mayores tasas de incidencia de DH $(88,99 / 100.000$ hbts.), al igual que Caquetá (99,00/100.000 hbts.). Mientras que Guainía, Guaviare y Vichada presentaron las incidencias más altas para este año.

En el 2008 se notificaron 32.797 casos de DC y 4.348 casos de DH para un total de 37.145 casos (Figura 1). Se observó una disminución en el número de casos de dengue registrados hasta la semana 52 de 2008, comparado con los del año 2007. Los departamentos que reportaron un mayor número de casos de DC fueron Atlántico con 4.534, Santander con 3.474, Norte de Santander con 3.151, Meta con 2.804 y Antioquia con 2.115. Igualmente, el mayor número de casos de DH se presentó en los departamentos de Santander con 1.350 casos, Norte de Santander con 751, Huila con 584 y Valle del Cauca con 310. La tasa de incidencia global de dengue fue de 183/100.000 hbts., (dengue clásico 161/100.000 hbts., y dengue hemorrágico 21/100.000 hbts.).

A la semana epidemiológica 52 de 2009 se notificaron 60.915 casos de DC, entre probables y confirmados, y 10.164 casos de DH, para un total de 71.079 casos de dengue reportados (Figura 1). En cuanto al comportamiento de la notificación, tanto de DC como DH, se observó un fuerte incremento con respecto al año anterior. Lo más sorprendente del 2009 fue el gran incremento de casos de DC reportados en el Valle del Cauca; pasó de 1.861 casos reportados en el 2008, a 12.117 casos en el 2009. Es decir, hubo un incremento de más del $651 \%$. Así mismo se presentó un fuerte incremento en Santander, con 6.675 casos; Norte de Santander con 5.179, Huila con 4.694, Antioquia con 4.566, Arauca con 3.311 y Boyacá con 3.160 casos. A pesar de que el Valle del Cauca reportó el mayor número de casos de dengue, no fue el departamento con mayor número de casos de DH; sólo se reportó 1.455. La lista la encabeza Santander con 2.816 casos, Norte de Santander con 1.931, Huila con 1.923 y Casanare con 441 casos. 2009 constituye el año con mayor número de casos de $\mathrm{DH}$ reportado en el país (Figura 1).

En el 2010 Colombia vivió la más grande epidemia de dengue de los últimos 11 años (Figura 1). El incremento fue drástico: de 9.745 casos reportados en 1992, pasó a 151.774 en el 2010. Es de anotar que teniendo en cuenta la nueva clasificación de dengue de la OMS, en el 2010 los casos de dengue ya no se reportaron como DC o DH, sino como dengue (D) y dengue grave (DG). Hasta 2009, Antioquia no estaba entre los departamentos con mayor número de casos de dengue reportados, pero pasó de 4.566 casos reportados en el 2009, a 25.527 en el 2010, indicando que el incremento fue de aproximadamente $600 \%$. El número de casos de DG en dicho departamento fue de 300. En el Valle del Cauca continuó la tendencia al incremento ya observada en el 2009, y pasó de 12.117 a 19.511 casos de dengue en el 2010. Santander pasó de 6.675 casos de DC reportados en el 2009, a 17.611 casos de dengue. Posiblemente el fenómeno más sorprendente se presentó en Risaralda, ya que en dicho departamento se había observado una tendencia a la disminución desde 2007; pasó de 490 casos de DC reportados en el 2009, a 11.623 en el 2010, lo que indica que el incremento fue de aproximadamente $2.370 \%$. También se observó un fuerte incremento en los departamentos de Tolima con 10.039 casos, Quindío con 9.753, Norte de Santander con 7.388 y Huila con 6.699 casos. A pesar del fuerte incremento de casos de dengue reportados, el número de casos de DG notificados no fue muy alto; el Valle del Cauca presentó 1.230 casos y fue el departamento con un mayor número de casos; luego está Huila con 976, Norte de Santander con 695 y Santander con 540 casos. Quindío fue el departamento con mayor incidencia de dengue con 2.059 casos/100.000 hbts., mientras que Huila lo fue para DG con 197 casos/100.000 hbts.

A la semana epidemiológica 52 de 2011 se notificó a SIVIGILA un total de 30.694 casos, 29.391 (96\%) casos de dengue y 1.303 (4\%) casos de dengue grave. Se observa un descenso de $80 \%$ en los casos de dengue notificados el año 2011 (30.694) comparado con el año epidémico 2010 (157.202). El 66\% de los casos de dengue reportados en el año 2011 proceden de 10 entidades territoriales: Meta 9\%, Valle 9\%, Norte de Santander 9\%, Tolima 8\%, 
Santander 7\%, Antioquia 7\%, Huila 6\%, Sucre 4\%, Cesar 4\% y Quindío 4\%. En DG el 76\% de los casos reportados proceden de 10 entes territoriales: Huila 19\%, Cesar 9\%, Valle 9\%, Santander 9\%, Norte de Santander $8 \%$, Córdoba $6 \%$, Meta $6 \%$, Antioquia 4\%, Casanare 3\% y Caquetá 3\%. Durante el año 2011 todos los entes territoriales presentaron disminución de casos de dengue y dengue grave respecto al 2010. Después de un fuerte incremento de casos de dengue reportados en el 2010, 2011 constituye el año con el menor número de casos de DG. A pesar de que existe mucha controversia sobre el reporte de casos de dengue en el departamento del Amazonas, 2011 fue el año con más casos reportados en dicha región del país: 527 casos de dengue y 3 muertos.

Hasta la semana epidemiológica $\mathrm{N}^{\circ} 51$ de 2012 se han notificado 53.141 casos totales de dengue, 51.724 (97\%) de dengue y 1.417 (3\%) dengue grave. Comparando con el año 2011 se documenta un aumento de casos distribuidos así: 29.977 casos totales de dengue equivalentes a un aumento de $43,5 \%$, de los cuales 28.694 casos corresponde a dengue (aumento 44,5\%) y dengue grave 1.283 casos (aumento 9,4\%). La incidencia acumulada de dengue hasta semana epidemiológica 51 es de 267,6 por 100.000 habitantes. E1 75,2\% de los casos de dengue proceden de 10 departamentos: Huila (11,6\%), Meta (11,5\%), Santander (9\%), Tolima (9\%), Cesar $(8,4 \%)$, Norte de Santander $(7,6 \%)$, Valle (6,2\%), Casanare (3,9\%), Antioquia (4,2\%), Sucre $(3,8 \%)$.

Hasta la semana epidemiológica 48 de 2013 se han notificado al Sistema de Vigilancia en Salud Pública del INS: 119.856 casos totales de dengue, 116.877 (97,5\%) de dengue y $2.929(2,5 \%)$ de dengue grave. Con corte a semana 48 de 2012 se había notificado 48.615 casos de dengue, que comparado con el 2013 corresponde a un aumento de $146 \%$. La incidencia acumulada nacional de dengue a la semana 48 es de 453,5 casos/100.000 hbts., cuya población a riesgo corresponde a la población urbana del país. La letalidad por dengue grave para Colombia se encuentra en $4,7 \%$.

Al analizar la distribución del DC por grupos de edad, se encontró que la mayoría de los casos se presentaron en el grupo de 15 a 45 años, pero el mayor porcentaje de casos de DH se observó en la población infantil o menores de 15 años. Esto podría también explicar la alta mortalidad en niños. Estos resultados sugieren que la población más susceptible a la infección por DENV son los niños, adolescentes y adultos jóvenes.

Otro aspecto que se evaluó fue el número de muertes ocasionadas por el dengue en Colombia, entre los años 2004 y 2013 (Figura 2). Como se puede observar, el mayor número de muertes se presentó en el 2010, con 233 decesos; a pesar de que en el 2011 se reportó un bajo número de casos de dengue, se presentaron 203 muertes; le sigue el 2011 con 2012 con 191, y 2005 y 2006 , con 47 muertes

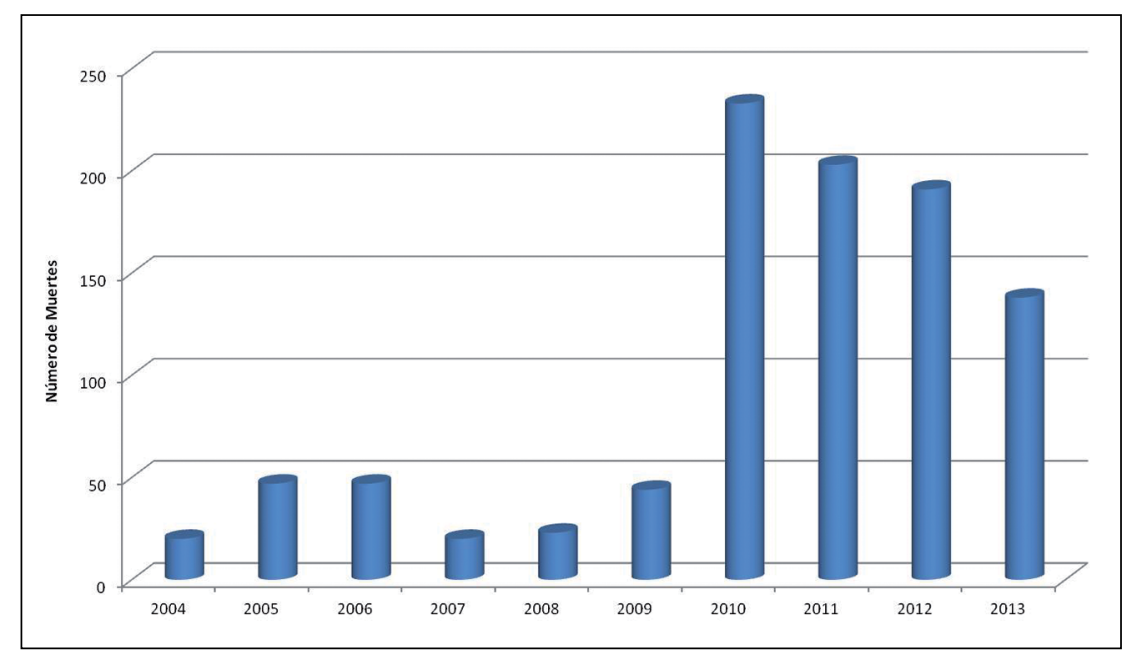

Figura 2. Reporte de casos de muerte por dengue en Colombia entre 2004-2013, según la base de datos de SIVIGILA y del INS.

cada año. Para el 2013 hasta la semana epidemiológica 48 se reportaron 138 muertes por dengue.

\section{Discusión}

El comportamiento de casos de dengue reportados en Colombia, en el período de 2004 a 2013 (Figura 1), indica que la infección por DENV presenta un comportamiento cíclico, el que muy posiblemente se repite cada tres o cuatro años. Además, 2010 fue el año con mayor registro de eventos relacionados con esta enfermedad, con más de 150.000 casos de DC, seguido del año 2002, en el que se notificaron más de 70.000 casos. Con respecto a $\mathrm{DH}$, el mayor número se reportó en el 2009, con un número aproximado de 10 mil casos (Figura 1).

La emergencia y re-emergencia de dengue en Colombia puede ser atribuida a un gran número de causas. Cambios sociales y demográficos, como el crecimiento poblacional y la urbanización sin planeación, resultan en el hacinamiento de comunidades que viven en centros urbanos con viviendas deficientes e inadecuados sistemas de proporción de agua, alcantarillado y manejo de residuos. Si a estos factores le sumamos el incremento en la migración de individuos a áreas endémicas del mosquito vector, el deterioro de las medidas de control y prevención del mosquito y los limitados recursos económicos y humanos destinados a la infraestructura de salud pública, es claro entender la razón por la cual el dengue se establece como una enfermedad persistente en una población ${ }^{2}$. Nuestro análisis muestra que en Colombia, los casos de dengue no se centran en una sola región o departamento; por tal razón es muy difícil poder establecer si existe una relación 
Figura 3. Regiones más afectadas por el dengue en Colombia.

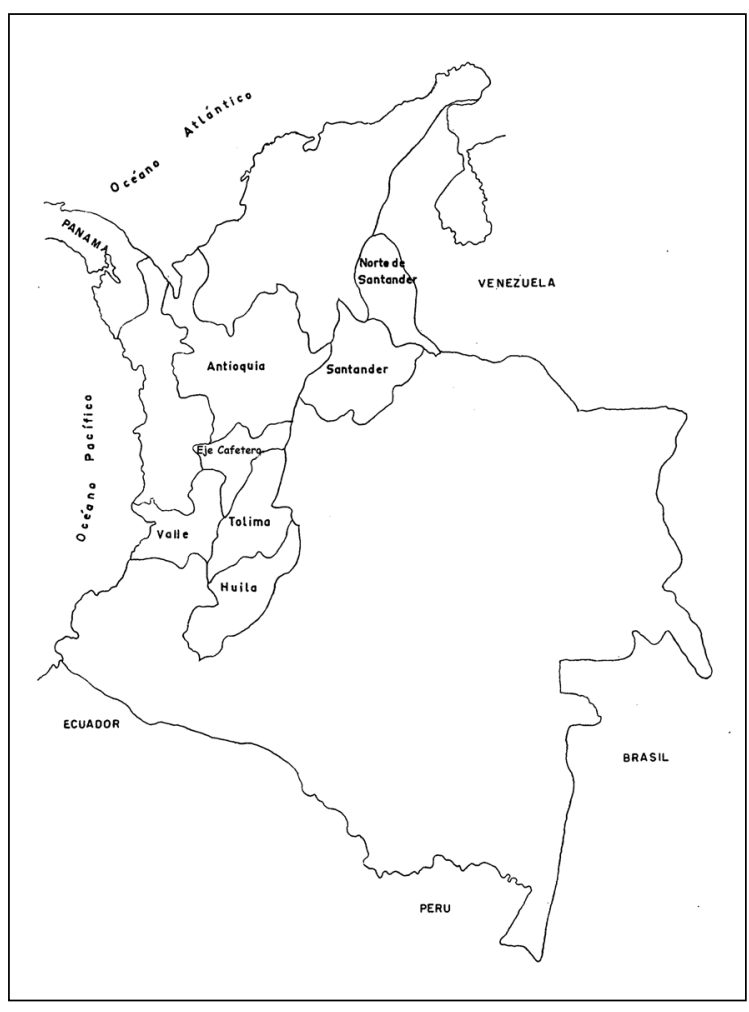

entre los casos de dengue y los índices de desarrollo y condiciones de vida de cada región. Así por ejemplo, los departamentos más afectados son Santander y Norte de Santander, los que, en términos generales, presentan unos índices de desarrollo y condiciones de vida inferiores al resto del país. Pero el dengue también se presenta en Antioquia, Valle del Cauca y algunas regiones de la zona cafetera (Quindío y Risaralda), cuyos habitantes presentan mejores condiciones de vida (Figura 3).

Igualmente, se ha reportado una estrecha relación entre el dengue y cambios climáticos globales. Por ejemplo, el incremento de casos de dengue se ha asociado con el Fenómeno del Niño, el que se caracteriza por grandes sequías y un aumento de la temperatura en todo el territorio. En consecuencia, esto conlleva a que los pobladores tengan que establecer estrategias para el almacenamiento de agua, pero a veces lo hacen de manera inapropiada. Este mismo tipo de asociación también se ha establecido en países como Guyana Francesa, Indonesia, Surinam y algunas islas del Pacífico, en los que se ha reportado epidemias de dengue, a consecuencia del incremento de la temperatura y disminución de las precipitaciones durante los años de El Niño ${ }^{12-14}$. Esos cambios ambientales son propicios para la proliferación de vectores asociados a enfermedades como el dengue, debido a que cambios en la temperatura tienden a afectar su dinámica de transmi- sión. Por ejemplo, temperaturas más cálidas reducen el tamaño de las larvas de $A$. aegypti afectando, en última instancia, el tamaño del adulto $^{15}$. El problema, tal como lo reportan los autores, es que los adultos más pequeños tienen que alimentarse con más frecuencia para desarrollar un lote de huevos, lo que aumenta la frecuencia de picaduras y con ello, un aumento en la transmisión del virus. Además, el desarrollo viral también se altera con el aumento de la temperatura debido a que el período de incubación extrínseco se reduce con temperaturas más altas, aumentando la proporción de mosquitos infectados en un momento dado. Es necesario anotar que para que la transmisión del virus ocurra, la hembra infectada necesita de un período de incubación extrínseco, que generalmente dura de 8 a 12 días, tiempo necesario para que el mosquito se vuelva infectante; sólo así el mosquito puede transmitir el DENV por picaduras a un individuo susceptible ${ }^{16}$. Por ejemplo, se ha demostrado que el período de incubación extrínseco para el DENV tipo 2 es de 12 días a $30^{\circ} \mathrm{C}$, pero se reduce a sólo 7 días a una temperatura de 32 a $35^{\circ} \mathrm{C}$, lo que significa un aumento de hasta tres veces en la transmisión de la enfermedad ${ }^{17}$. Dicho comportamiento sugiere que temperaturas ligeramente más altas dentro del rango de la viabilidad del vector del DENV estimulan a los mosquitos infectados a picar con más frecuencia, causando un mayor riesgo de infección en hospederos susceptibles. Además, un aumento de las precipitaciones, que generalmente suceden durante el fenómeno de El Niño, lleva a un incremento de los sitios de reproducción de los mosquitos y a un aumento de la supervivencia de su estado adulto, promoviendo de esta forma la incidencia de dengue durante esos períodos ${ }^{15}$. En el presente estudio no se encontró relación estadística entre la dinámica de transmisión de dengue en Colombia y algunas variables climáticas como temperatura, precipitación y humedad relativa. Sin embargo, se observó una relación entre el aumento de la incidencia de la enfermedad en el año 2007, con la precipitación y temperatura promedio en algunos departamentos de Colombia (Figuras 4 y 5). Se observa que departamentos como Caquetá, Guainía, Guaviare y Meta, que presentaron altas incidencias de dengue en el año 2007 son departamentos que presentan precipitaciones mayores o iguales a $3.000 \mathrm{~mm}$ de agua al año (Figura 4). Además, es evidente que los departamentos que presentan mayor incidencia de dengue son los que presentan temperaturas promedio mayores a los $25^{\circ} \mathrm{C}$ (Figura 5). A pesar de que no se observó significancia estadística entre las variables climáticas estudiadas y la incidencia de dengue, debe considerarse que el clima juega un papel importante en la distribución espacio temporal de la incidencia de la enfermedad, atribuyendo, como consecuencia directa o indirecta del aumento en la incidencia de la enfermedad, a variaciones en la humedad, precipitación y/o a las altas temperaturas. Es posible que 

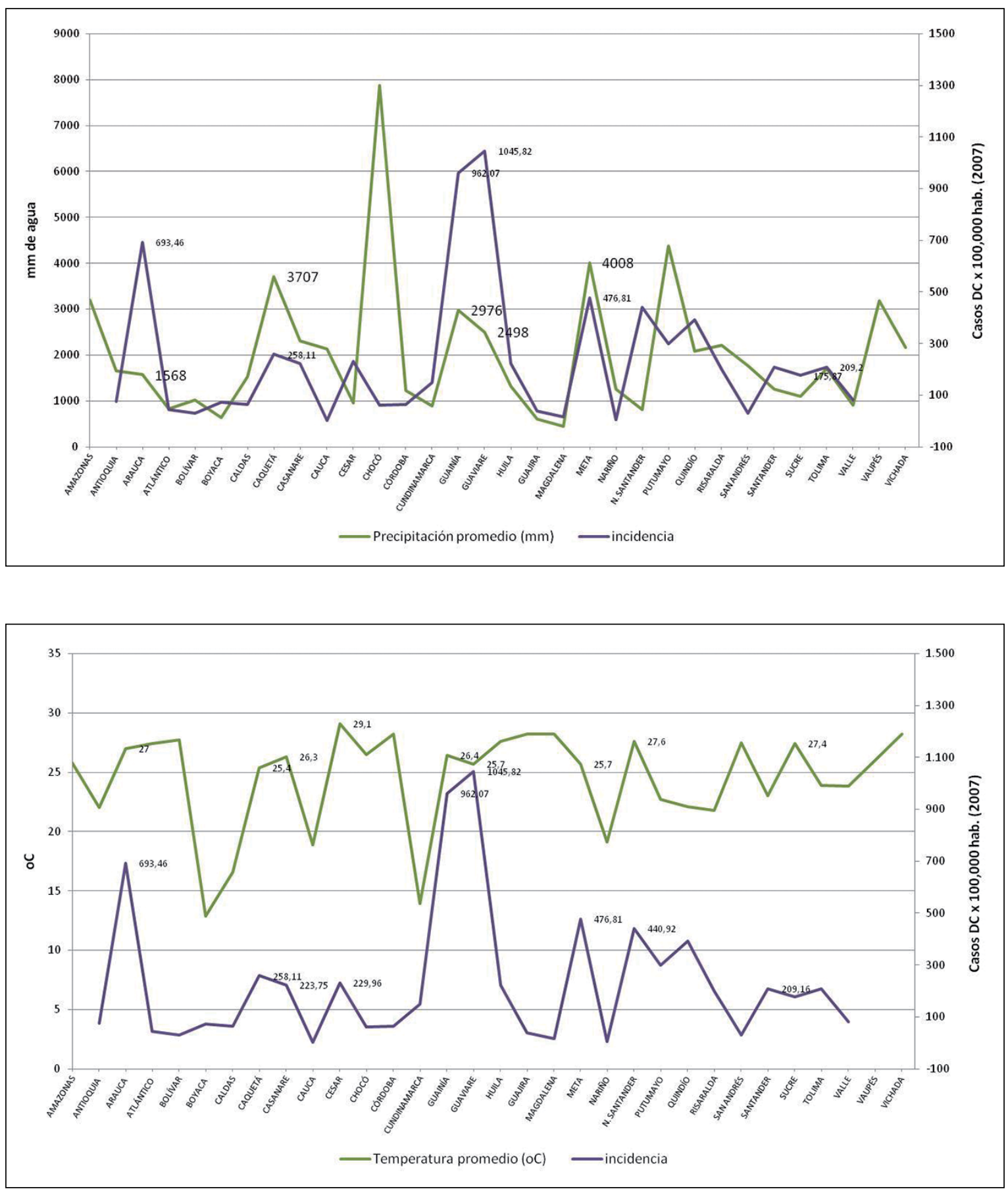

no se haya encontrado tal relación significativa debido a que es necesaria la búsqueda de más datos y variables, ya que también debe considerarse que la transmisión de dengue es multicausal, donde otros elementos como las características sociales, culturales y económicas de la población pueden influir en la dinámica de esta compleja enfermedad.
En Colombia, todos los episodios de El Niño hasta el año 1998, excepto el de 1993, se asociaron con una incidencia de dengue ${ }^{12}$. En general, el dengue se ha comportado como una enfermedad endémica, con brotes epidémicos cíclicos, en casi todas las poblaciones por debajo de los 1.800 metros sobre el nivel del mar, lo que equivale a $900.000 \mathrm{~km}^{2}$ de los $1.138 .914 \mathrm{~km}^{2}$ de extensión
Figura 4. Asociación entre la incidencia de dengue clásico en el 2007 y la precipitación media por departamento.
Figura 5. Asociación entre la incidencia de dengue clásico en el 2007 y la temperatura media por departamento. 
del país y donde viven aproximadamente 20.000.000 de personas. Aedes aegypti es el principal transmisor del dengue en Colombia, y se encuentra distribuido en casi $80 \%$ del territorio, situado entre 1.000 a 2.200 metros sobre el nivel del mar ${ }^{18}$. Esta especie de mosquitos es el principal vector del virus en todo el mundo y está muy bien adaptada a la vida urbana, donde se reproducen en aguas estancadas generadas por el hombre ${ }^{19}$. Estas especies permanecen activas durante el día, pero generalmente se alimentan temprano en la mañana o al atardecer ${ }^{20}$. Consecuencia del cambio climático, entre otros factores, es posible encontrar el $A$. aegypti en municipios localizados alrededor de los $2.000 \mathrm{msnm}$, lo cual se ha convertido en un gran problema ya que esto involucra un gran número de municipios $^{21}$. Es así como en 125 municipios localizados en 22 departamentos del país se ha detectado índices aédicos (IA) mayores de 35\%. En municipios como Puerto Colombia (Atlántico), Puerto Boyacá (Boyacá), Dorada (Caldas), Girardot (Cundinamarca), Ciénaga (Magdalena), Tumaco (Nariño), Villavicencio (Meta), San Andrés (San Andrés), Arauca (Arauca), Florencia (Caquetá) y Cartagena; además, los índices de Breteau son mayores de $30^{22}$. Es necesario aclarar que normalmente se emplean tres índices para registrar los niveles de infestación por mosquitos: el índice de infestación domiciliaria o $\mathrm{IH}$, el índice de contenedores o recipientes (IC) y el índice de Breteau (IB). IH indica el porcentaje de casas positivas para larvas de Aedes, IC el porcentaje de contenedores positivos para las larvas de Aedes, y IB indica el número de recipientes positivos para Aedes, por cada 100 casas estudiadas $^{23}$.

\section{Conclusiones}

Nuestro análisis retrospectivo muestra que en Colombia los brotes de dengue son cíclicos y son reportados principalmente en departamentos del Norte de Santander, Santander, Huila, Tolima, Valle del Cauca y Antioquia (Figura 3). A pesar que se ha reportado la existencia de los cuatro serotipos de DENV, ninguno de ellos es dominante, aunque el más frecuente es DENV2. Posiblemente, consecuencia del cambio climático o al índice de desarrollo y condiciones de vida, los casos de dengue han aumentado drásticamente desde que se describió el primer caso en Colombia, en diciembre de 1989, en Puerto Berrío (Antioquia). En consecuencia, la pérdida económica puede llegar a millones de dólares anualmente. De ahí la necesidad de realizar campañas eficaces en la lucha contra el vector en zonas de alta densidad del vector y en las regiones donde se reporta los mayores casos de dengue, antes y después del Fenómeno del Niño, para frenar la amenaza del dengue. Igualmente es necesario establecer programas de educación que orientan la población más vulnerable sobre la enfermedad, con el fin de generar conocimientos que permitan una consulta oportuna. Finalmente se debe aumentar el recurso humano y económico destinado a la infraestructura de salud pública de tal manera que se pueda mejorar el diagnóstico y el acceso a los servicios de salud. Esto es de suma importancia si se tiene en cuenta que la mortalidad por dengue es evitable en $98 \%$ de los casos, ya que los aspectos encontrados como críticos en la atención al paciente, han sido frecuentemente la falta de conocimientos de la población, lo que impide una consulta oportuna, además de las fallas en el diagnóstico y las barreras de acceso a los servicios de salud en Colombia.

Agradecimientos. A Colciencias y al grupo de Inmunovirología.

\section{Resumen}

Introducción: El dengue es un importante problema de salud pública mundial para el cual aún no existe una vacuna, por lo que el aporte al conocimiento de su comportamiento es útil para la ejecución de estrategias adecuadas para su control. Objetivo: Analizar la incidencia de dengue en los últimos diez años en Colombia (2004-2013), resaltando los períodos y regiones en las que se reporta el mayor número de casos. Métodos: Se realizó una búsqueda de casos de dengue reportados en Colombia entre 2004 y 2013 en registros del SIVIGILA y el INS. Las variables meteorológicas se obtuvieron del IDEAM y su correlación con la incidencia de dengue se halló por el método de correlación de Pearson. Resultados: Nuestro análisis muestra que cada año se presenta un incremento en el número de casos y las regiones más afectadas son Santander, Norte de Santander, Huila, Tolima y Valle del Cauca. El 2010 fue el año con mayor registro de eventos, con más de 150.000 casos. Discusión: Los resultados indican que la infección por DENV presenta un comportamiento cíclico, que muy posiblemente se repite cada tres o cuatro años y dicha ocurrencia de casos puede ser atribuida a cambios sociales y climáticos.

\section{Referencias bibliográficas}

1.- Guzmán M G, Kouri G. Dengue and dengue hemorrhagic fever in the Americas: lessons and challenges. J Clin Virol 2003; 27 (1): 1-13.

2.- Gubler D J. Epidemic dengue/dengue hemorrhagic fever as a public health, social and economic problem in the 21 st century. Trends Microbiol 2002; 10 (2): 100-3.

3.- Weaver S C, Vasilakis N. Molecular evolution of dengue viruses: Contributions of phylogenetics to understanding the history and epidemiology of the preeminent arboviral disease. Infect Genet Evol 2009; 9: 523-40.

4.- Mairuhu A T, Wagenaar J, Brandjes D P, van Gorp E C. Dengue: an arthropod-borne disease of global importance. Eur J Clin Microbiol Infect Dis 2004; 23(6): 425-33. 
5.- $\quad$ Ligon B L. Dengue fever and dengue hemorrhagic fever: a review of the history, transmission, treatment, and prevention. Semin Pediatr Infect Dis 2005; 16 (1): 60-5.

6.- Halstead S B. Dengue. Lancet 2007; 370: 1644-52.

7.- WHO. Global Alert and Response: Impact of Dengue [Documento en internet] 2006 [Acceso en febrero de 2013]. Disponible en: www.who. int/csr/disease/dengue/impact/en/index.html.

8.- WHO. Dengue: Guidelines for diagnosis, Treatment, Prevention and Control [Documento en internet] 2009 [accedido febrero de 2013]. Disponible en: www.who.int/topics/dengue/en/.

9.- OPS. Información y Conocimiento en Salud [Documentos en internet] [accedido en noviembre de 2012]. Disponible en: www.col ops-oms.org/sivigila/IndiceBoletines2000.asp.

10.- INS. Vigilancia y Control en Salud Pública [Documeno en internet] [accedido en diciembre de 2012]. Disponible en: www.ins.gov. co/?idcategoria $=89637$.

11.- IDEAM. Atlas climatológico de Colombia [Documeno en internet] 2005 [accedido en enero de 2013]. Disponible en: https:// documentacion.ideam.gov.co/openbiblio/ Bvirtual/019711/019711.htm.

12.- Gagnon A, Bush A, Smoyer-Tomic K. Dengue epidemics and the El Niño Southern Oscillation. Clim Res 2001; 19: 35-43.

13.- Hales S, Weinstein P, Woodward A. Dengue fever epidemics in the South Pacific: driven by El Nino Southern Oscillation? Lancet 1996; 348 (9042): 1664-5.

14.- Hales S, Weinstein P, Souares Y, Woodward A. El Nino and the dynamics of vectorborne disease transmission. Environ Health Perspect 1999; 107 (2): 99-102.

15.- Sehgal R. Dengue fever and El Nino. Lancet 1997; 349 (9053): 729.

16.- McBride W J, Bielefeldt-Ohmann H. Dengue viral infections; pathogenesis and epidemiology. Microbes Infect 2000; 2 (9): 1041-50.

17.- Watts D M, Burke D S, Harrison B A, Whitmire R E, Nisalak A. Effect of temperature on the vector efficiency of Aedes aegypti for dengue 2 virus. Am J Trop Med Hyg 1987; 36 (1): 143-52.
18.- SIVIGILA. Hechos en Vigilancia en Salud Pública. Disponible en: www.col.ops-oms.org/sivigila/2001/ BOLE52_2001.htm.

19.- Wilder-Smith A, Gubler D J. Geographic expansion of dengue: the impact of international travel. Med Clin North Am 2008; 92 (6): 1377-90, x.

20.- Rigau-Pérez J G, Clark G G, Gubler D J, Reiter P, Sanders E J, Vorndam A V. Dengue and dengue haemorrhagic fever. Lancet 1998; 352 (9132): 971-7.

21.- Ríos J F. Aspectos entomológicos del dengue. Infectio 2004; 8 (3): 231-5.

22.- SIVIGILA. Boletín Epidemiológico Semanal. Disponible en: www.col.ops-oms.org/ sivigila/2002/BOLE52_02.htm.

23.- Thavara U, Tawatsin A, Chansang C, Kong-ngamsuk W, Paosriwong S, Boon-Long J, et al. Larval occurrence, oviposition behavior and biting activity of potential mosquito vectors of dengue on Samui Island, Thailand. J Vector Ecol 2001; 26 (2): 172-80. 